\title{
On Complex Economic Dynamics: Agent-Based Computational Modeling and Beyond
}

\author{
Shu-Heng Chen, Ye-Rong Du, Ying-Fang Kao, Ragupathy Venkatachalam, 4 \\ and Tina Yu
}

\begin{abstract}
This chapter provides a selective overview of the recent progress in the 6 study of complex adaptive systems. A large part of the review is attributed to agent- 7 based computational economics (ACE). In this chapter, we review the frontier of 8 ACE in light of three issues that have long been grappled with, namely financial 9 markets, market processes, and macroeconomics. Regarding financial markets, 10 we show how the research focus has shifted from trading strategies to trading 11 institutions, and from human traders to robot traders; as to market processes, we 12 empathetically point out the role of learning, information, and social networks 13 in shaping market (trading) processes; finally, in relation to macroeconomics, we 14 demonstrate how the competition among firms in innovation can affect the growth 15 pattern. A minor part of the review is attributed to the recent econometric computing, 16 and methodology-related developments which are pertinent to the study of complex 17 adaptive systems.

Keywords Financial markets - Complexity thinking · Agent-based

This book is the post-conference publication for the 21st International Conference 21 on Computing in Economics and Finance (CEF 2015) held on June 20-22, 201522 in Taipei. Despite being the largest conference on computational economics for 23 two decades, CEF has never produced any book volume that documents the path- 24 breaking and exciting developments made in any of its single annual events. 25

S.-H. Chen $(\bowtie) \cdot$ Y.-F. Kao $\cdot$ T. Yu

AI-ECON Research Center, Department of Economics, National Chengchi University, Taipei,

Taiwan

Y.-R. Du

Taiwan Institute of Economic Research (TIER), Taipei, Taiwan

R. Venkatachalam

Institute of Management Studies, Goldsmiths, University of London, London, UK 
For many years, the post-conference publications had always been in the form of 26 journals' special issues, which, unfortunately, have ceased to continue in recent 27 years. Consequently, although the voices of CEF had been loud and clear for 28 many years on many prominent issues, they may have been forgotten as time goes 29 by. Without proper archives, it will be difficult for the new-comers to trace the 30 important contributions that the conferences have made in the field of computational 31 economics.

Two years ago, Springer launched a new series, Springer Proceedings in 33 Complexity, to publish proceedings from scholarly meetings on topics related to the 34 interdisciplinary studies of the science of complex systems. The scope of CEF fits 35 the mission of this series perfectly well. Not only does CEF deal with problems 36 which are sufficiently complex to defy an analytical solution from Newtonian 37 Microeconomics [9], but CEF methods also treat economics as a science of 38 complex systems, which requires complexity thinking both in terms of ontology and 39 epistemology [22]. Therefore, when Christopher Coughlin, the publishing editor 40 of the series, invited us contribute a volume, we considered it to be a golden 41 opportunity to archive the works presented at CEF 2015, in a way similar to what 42 we had done previously in the form of journals' special issues. 43

However, CEF 2015 had a total of 312 presentations, which covered many 44 aspects of CEF. To include all of them in a single volume is doubtlessly impossible. 45 A more practical alternative would be to select an inclusive and involving theme, 46 which can present a sharp focus that is neither too narrow nor too shallow. It is 47 because of this consideration that we have chosen one of the most active areas 48 of CEF, namely agent-based computational economics (ACE), as the main theme 49 of this book and have included ten chapters which contribute to this topic. These 50 ten chapters are further divided into three distinct but related categories: financial 51 markets, market processes and the macroeconomy. Although there are other areas of 52 ACE that have also made important advances, we believe that without tracking the 53 development of these three research areas, the view of ACE will become partial or 54 fragmented. These ten chapters, constituting the first part of the book, will be briefly 55 reviewed in Sect. 1.

In addition to these ten chapters, we include three chapters that present new 57 methodologies and technologies to study the complex economic dynamics. Three 58 chapters are contributions of this kind. The first one is an econometric contribution 59 to the identification of the existence and the extent of financial integration. The 60 second one addresses the role of supercomputers in developing large-scale agent- 61 based models. The last one challenges the capability of formal reasoning in 62 modeling economic and financial uncertainties. It also advocates a reform of the 63 economic methodology of modeling the real-world economy. These three chapters, 64 constituting the second part of the book, will be briefly reviewed in Sect. 2. 


\section{Agent-Based Computational Economics}

One core issue that ACE seeks to address in economics is how well the real economy 67 performs when it is composed of heterogeneous and, to some extent, boundedly 68 rational and highly social agents. In fact, a large body of published ACE works can 69 be connected to this thread. This issue is of particular importance when students of 70 economics are nowadays still largely trained under Newtonian economics using the 71 device of a representative agent who is assumed to be fully rational in seeking to 72 maximize a utility function. As an alternative research paradigm to the mainstream, 73 ACE attempts to see how our understanding of the economy can become different 74 or remain the same when these simplifications are removed.

Part of ACE was originated by a group of researchers, including Brian Arthur, 76 John Holland, Blake LeBaron, Richard Palmer, and Paul Tayler, who developed an 77 agent-based model called the Santa Fe Artificial Stock Market to study financial 78 markets [1,5]. Quite interestingly, their original focus was not so much on the 79 financial market per se, i.e., the financial market as an institutional parameter, 80 but on the exploration of trading strategies under evolutionary learning, the co- 81 evolution of trading strategies and the emergence of market price dynamics. This 82 focus drove the early ACE research away from the role of trading mechanisms and 83 institutional arrangements in the financial markets, which was later found to be a 84 substantially important subject in computational economics and finance. Section 1.185 will summarize the three ACE works that focus on trading institutions, rather than 86 trading strategies.

Market process theory investigates how a market moves toward a state of general 88 economic equilibrium and how production and consumption become coordinated. 89 Agent-based modeling is a modern tool used to analyze the ideas associated with 90 a theoretical market process. In Sect. 1.2, we will give an overview of six works 91 that investigate the price discovery process, market dynamics under individual, and 92 social learning and market herding behaviors using agent-based simulation. 93

Macroeconomics studies the performance, structure, behavior, and decision- 94 making of an economy as a whole. ACE is a modern methodology that is applied to 95 examine the macroeconomy. In Sect. 1.3, we introduce the work using ACE models 96 to analyze the macroeconomic dynamics under product innovation.

\subsection{Financial Markets}

Dark Pools is an alternative trading institution to the regular exchanges that have 99 gained popularity in recent years. In dark pools trading, there is no order book 100 visible to the public; hence the intention of trading is not known until the order 101 is executed. This provides some advantages for the institutional traders who can 102 obtain a better realized price than would be the case if the sale were executed on a 103 regular exchange. However, there are also disadvantages in that the order may not 104 
be executed because of the lack of information for the order's counter parties. With 105 the growing usage of dark pools trading, concerns have been raised about its impact 106 on the market quality. In the chapter, entitled "Dark Pool Usage and Equity Market 107 Volatility," Yibing Xiong, Takashi Yamada, and Takao Terano develop a continuous 108 double-auction artificial stock market that has many real-world market features. 109 Using various institutional parametric setups, they conduct market simulations to 110 investigate the market's stability under dark pools trading.

The institutional-level parameters that they investigated are:

- Dark pool usage probability $(0,0.2$ or 0.4$)$;

- Market-order proportion (0.3-0.8);

- Dark pool cross-probability (0.1-1.0), which is the probability that the buy orders 115 and sell orders in the dark pool are being crossed at the mid-price of the exchange. 116 A lower cross-probability indicates a relatively longer order execution delay in 117 the dark pool.

Their simulation results indicated that the use of mid-price dark pools decreases market volatility, which makes sense because the transaction is not visible to the public until the order is completed. The transactional impact on the market stock prices is therefore minimized. Moreover, they found that the volatility-suppressing effect is stronger when the dark pool usage is higher and when the market-order proportion submitted to the dark pool is lower. ${ }^{1}$ They also reported that the dark pool cross-probability did not have any effects on the market volatility.

119 120 121 122 123 124

Another trend in recent financial markets is the use of computer algorithms 126 to perform high frequency trading (HFT). Since computer programs can execute 127 trades much faster than humans, stocks and other instruments exhibit rapid price 128 fluctuations (fractures) over sub-second time intervals. One infamous example is 129 the flash crash on May 6, 2010 when the Dow Jones Industrial Average (DJIA) 130 plunged by around 7\% (US\$1 trillion) in $5 \mathrm{~min}$, before recovering most of the fall 131 over the following $20 \mathrm{~min}$. To understand the impact of HFT on financial markets, 132 in the chapter, entitled "Modelling Complex Financial Markets Using Real-Time 133 Human-Agent Trading Experiments," John Cartlidge and Dave Cliff used a real- 134 time financial-market simulator (OpEx) to conduct economic trading experiments 135 between humans and automated trading algorithms (robots). 136

The institutional-level parameters that they investigated included: 137

- Robots' trading speed, which is controlled by the sleep-wake cycle $\left(t_{s}\right)$ of robots. 138 After each decision (buy, sell, or do nothing) is made, a robot will sleep for $t_{S} 139$ milliseconds before waking up to make the next decision. The smaller that $t_{S}$ is, 140 the faster the robots' trading speed and the higher their trading frequency. 141

- Cyclical vs. random markets: In each experiment, there are six pre-generated 142 assignment permits, each of which contains a permit number and a limit price- 143 the maximum value at which to buy, or the minimum value at which to sell. 144

\footnotetext{
${ }^{1}$ See [16] for similar findings using empirical data.
} 
The lower the permit number is, the farther away the limit price is from the 145 equilibrium. In a cyclical market, the permits are issued to humans and robots 146 following the permit numbers. By contrast, the permits are issued in random 147 order in a random market.

Their simulation results showed that, under all robot and human market setups, 149 robots outperform humans consistently. In addition, faster robot agents can reduce 150 market efficiency and this can lead to market fragmentation, where humans trade 151 with humans and robots trade with robots more than would be expected by chance. 152 In terms of market type, the cyclical markets gave very different results from those 153 of random markets. Since the demand and supply in the real-world markets do not 154 arrive in neat price-ordered cycles like those in the cyclical markets, the results from 155 cyclical markets cannot be used to explain what happened in the real-world financial 156 markets. The authors used these two types of markets to demonstrate that, if we want 157 to understand complexity in the real-world financial markets, we should move away 158 from the simple experimental economic models first introduced in the 1960s. 159

In the chapter, entitled "Does High-Frequency Trading Matter?", Chia-Hsuan 160 Yeh and Chun-Yi Yang also investigated the impact of HFT on market stability, price 161 discovery, trading volume, and market efficiency. However, instead of conducting 162 real-time experiments using humans and robots, they developed an agent-based 163 artificial stock market to simulate the interaction between HFT and non-HFT agents. 164 In addition, unlike the robots in the previous chapter that used pre-generated permits 165 to submit buy and sell orders for price matching, the agents in this study are more 166 sophisticated in terms of using heuristics to make trading decisions. Moreover, the 167 agents have learning ability to improve their trading strategies through experiences. 168

In their agent-based model, the trading speed is implemented as the agents' 169 capability to process market information for decision-making. Although instant 170 market information, such as the best bid and ask, is observable for all traders, only 171 HFT agents have the capability to quickly process all available information and to 172 calculate expected returns for trading decisions. Non-HFT agents, however, only 173 have the capability to process the most recent $k$ periods' information. The smaller 174 that $k$ is, the greater the advantage that the HFT agents have over non-HFT agents. 175

The institutional-level parameters that they investigated include: 176

- The number of HFT agents in the market $(5,15,20)$; 177

- The activation frequency of HFT agents, which is specified by the number of 178 non-HFT agents $(m=40,20,10)$ that have posted their quotes before an HFT 179 agent can participate in the market. The smaller that $m$ is, the more active the 180 HFT agents are in participating in the trading.

Their simulation results indicated that market volatilities are greater when there 182 are more HFT agents in the market. Moreover, a higher activation frequency of the 183 HFT agents results in greater volatility. In addition, HFT hinders the price discovery 184 process as long as the market is dominated by HFT activities. Finally, the market 185 efficiency is reduced when the number of HFT agents exceeds a threshold, which is 186 similar to that reported in the previous chapter. 


\subsection{Market Processes}

The agriculture market in Luxembourg is thin, in terms of volume turnover, and 189 the number of trades in all commodities is small. While the information on market 190 products can be obtained through an annual survey of the farmers, the market 191 products trading price information is not accessible to the public. In the chapter, 192 entitled "Modelling Price Discovery in an Agent Based Model for Agriculture 193 in Luxembourg," Sameer Rege, Tomás Navarrete Gutiérrez, Antonino Marvuglia, 194 Enrico Benetto, and Didier Stilmant have proposed an agent-based model to 195 simulate the endogenous price discovery process under buyers and sellers who are 196 patient or impatient in submitting their bid/ask quotes.

In this model, agents are farmers whose properties (area, type, crops, etc.) are 198 calibrated using the available survey data. The model is then used to simulate a 199 market that contains 2242 farmers and ten buyers to trade 22 crops for four rounds. 200 In each round, after all buyers and farmers have submitted the quantity and price for 201 a commodity to buy or sell, the buyer who offers the highest price gets to purchase 202 the desired quantity. If only partial quantity is satisfied under the offered price, the 203 unmet quantity is carried over to the remaining rounds. Similarly, the sellers whose 204 products do not get sold under the offered price are carried over to the remaining 205 rounds. Based on the trading price in the initial round, buyers and sellers can adjust 206 their bid/ask prices in the remaining rounds to achieve their trading goals.

Some buyers/sellers are impatient and want to complete the trading in the next 208 round by increasing/decreasing the bid/ask prices to the extreme, while others are 209 more patient and willing to gradually adjust the prices during each of the remaining 210 three rounds. Based on their simulation, they found that the trading quantities and 211 prices produced by patient and by impatient traders have very different distributions, 212 indicating that traders' behaviors in submitting their bids/asks can impact the price 213 discovery process in an economic market.

In the chapter, entitled "Heterogeneity, Price Discovery and Inequality in an 215 Agent-Based Scarf Economy," Shu-Heng Chen, Bin-Tzong Chie, Ying-Fang Kao, 216 Wolfgang Magerl, and Ragupathy Venkatachalam also used an agent-based model 217 to investigate the price discovery process of an economic market. However, their 218 agents are different from those in the previous chapter in that they apply individual 219 and social learning to revise their subjective prices. The focus of this work is to 220 understand how agents' learning behaviors impact the efficacy of price discovery 221 and how prices are coordinated to reach the Walrasian equilibrium.

The model is a pure exchange economy with no market makers. Each agent has 223 its own subjective prices for the commodities and agents are randomly matched for 224 trading. The learning behavior of an agent is influenced by the intensity of choice 225 $\lambda$, which specifies the bias toward the better-performing prices in the past. When $\lambda 226$ is high, the agent trusts the prices that have done well (the prices can be from self ${ }_{227}$ and from other agents) and uses them to adjust its prices for the future trades. If $\lambda 228$ is low, the agent is more willing to take risk incorporating prices that have not done 229 well in the past for the future trades. 
Their simulation results showed that agents with a low $\lambda(0-3)$ have their 231 subjective prices converging close to the Walrasian equilibrium. This means risk- 232 taking agents are good at discovering prices toward the general equilibrium. 233 Moreover, some agents with a large $\lambda(>4)$ also have their market prices converging 234 to the general equilibrium. The authors analyzed those high $\lambda(>4)$ agents in more 235 detail and found those agents to also be imitators who copied prices that have done 236 well in the past to conduct most of their trades. This strategy enhanced their price 237 coordination toward the general equilibrium.

In terms of accumulated payoffs, the agents with low $\lambda(0-3)$ who also mixed 239 innovation and imitation in adjusting their subjective prices have obtained medium 240 or high payoffs. Meanwhile, the agents with high $\lambda(>4)$ who are also imitators have 241 received very high payoffs. Finally, the high $\lambda(>4)$ agents who are also reluctant 242 to imitate other agents' prices have received abysmal accumulated payoffs. Based 243 on this emerging inequality of payoffs, the authors suggested that different learning 244 behaviors among individuals may have contributed to the inequality of wealth in an 245 economy.

In the chapter, entitled "Rational Versus Adaptive Expectations in an Agent- 247 Based Model of a Barter Economy," Shyam Gouri Suresh also investigated market 248 dynamics under agents with learning ability in a pure exchange or barter economy. 249 In this direct exchange market, an agent can apply individual or social learning to 250 predict the productivity level of his next exchange partner. Based on the prediction, 251 the agent then decides his own productivity level. Under the individual learning 252 mode, the prediction is based on the productivity level of the agent's current 253 exchange partner while in the social learning mode, the prediction is based on the 254 productivity level of the entire population.

In this model, the productivity level of an agent can be either high or low and 256 there is a transition table that all agents use to decide their current productivity 257 level according to their previous productivity. Additionally, an agent can incorporate 258 his prediction about the productivity level of his next exchange partner to decide 259 his current productivity level. This prediction can be carried out through either 260 individual or social learning. Finally, to maximize his utility, an agent only adopts 261 high productivity when his transition table indicates high productivity and his next 262 exchange partner is also predicted to have high productivity.

The simulation results showed that the market per capita outputs or average 264 outputs converged to low productivity under individual learning. This is because 265 each time when an agent trades with another agent with low productivity, the agent 266 will decide to produce low outputs in the next period regardless of the productivity 267 specified by the transition table. This action in turn causes the agent he interacts 268 with in the next period to produce low outputs in the period subsequent to the next. 269 When an agent encounters another agent who has produced a high level of outputs, 270 the agent will only adopt high productivity in the next period if the transition table 271 also specifies high productivity. As a result, the market average outputs converge to 272 low productivity. 
By contrast, the market average outputs converge to high productivity under 274 social learning, when the population size is large (100 in their case). This is because, 275 in a large population, the likelihood of the population-wide distribution of produc- 276 tivity level being extreme enough to cause it to fall below the high-productivity 277 threshold is low. Consequently, as all agents started with high productivity, the 278 market average outputs remained high throughout the simulation runs. 279

In addition to the price discovery process and productivity level prediction, 280 traders' learning behaviors might have impacted the forward premium in the foreign 281 exchange market. In the chapter, entitled "Does Persistent Learning or Limited 282 Information Matter in the Forward Premium Puzzle?, Ya-Chi Lin investigated 283 whether the interactions between adaptive learning and limited market information 284 flows can be used to explain the forward premium puzzle.

The forward premium puzzle in the foreign exchange market refers to the well- 286 documented empirical finding that the domestic currency is expected to appreciate 287 when domestic nominal interest rates exceed foreign interest rates [4, 10, 14]. This 288 is puzzling because economic theory suggests that if all international currencies are 289 equally risky, investors would demand higher interest rates on currencies expected 290 to fall, and not to increase in value. To examine if investors' learning behaviors 291 and their limited accessibility to market information may explain this puzzle, Lin 292 designed a model where each agent can learn to predict the expected exchange rates 293 using either full information (day $t$ and prior) or limited information in the past (day 294 $t-1$ and prior). 295

In this model, the proportion of agents that have access to full information, $n$, is 296 an exogenous parameter. In addition, an agent has a learning gain parameter $\gamma$ that 297 reflects the learning strength. They simulated the model under different values of $n, 298$ from 0.1 to 1 , and $\gamma$, from 0.02 to 0.1 , and found that the forward premium puzzle 299 exists under small $n$ for all values of $\gamma$. Moreover, when agents were allowed to 300 choose between using limited or full information for forecasting, all agents switched 301 to using full information (i.e., $n=1$ ) and the puzzle disappeared for all values of 302 $\gamma$. This suggests that limited information might play a more important role than 303 learning in explaining the forward premium puzzle. However, regardless of the 304 values of $n$ and $\gamma$, the puzzle disappeared when tested in the multi-period mode. 305 This indicates that limited information alone is not sufficient to explain the puzzle. 306 There are other factors involved that will cause the puzzle to occur. 307

Herding is a well-documented phenomenon in financial markets. For example, 308 using trading data from US brokerages, Barber et al. [3] and Kumar and Lee [13] 309 showed that the trading of individual investors is strongly correlated. Furthermore, 310 based on trading data from an Australian brokerage, Jackson [12] reported that 311 individual investors moved their money in and out of equity markets in a systematic 312 manner. To macroscopically study the effects of herding behavior on the stock return 313 rates and on the price volatility under investors with different interaction patterns, 314 in the chapter, entitled "Price Volatility on the Investor's Social Network," Yangrui 315 Zhang and Honggang Li developed an agent-based artificial stock market model 316 with different network structures. 
In their interaction-based herding model, the trading decision of an agent 318 is influenced by three factors: (1) personal belief; (2) public information, and 319 (3) neighbors' opinions. Their work investigated the following institutional-level 320 parameters:

- Agents' interaction structures: regular, small-world, scale-free, and random 322 networks;

- Agents' trust in their neighbors' opinions (1-3);

Their simulation results showed that the market volatility is the lowest when 325 the agents are connected in a regular network structure. The volatility increases 326 when agents are connected under small-world or scale-free structures. The market 327 volatility is the highest when agents are connected under a random network 328 structure. This makes sense as the more irregular the agents' interaction pattern 329 is, the higher the price fluctuations and market volatility. In addition, they found 330 that the more an agent trusts in his neighbors' opinions, the greater the volatility of 331 the stock price. This is also expected, as the more weight an agent attaches to his 332 neighbors' opinions, the more diverse the trading decisions can be, and hence the 333 higher that the price volatility becomes.

In the chapter, entitled "The Transition from Brownian Motion to Boom- 335 and-Bust Dynamics in Financial and Economic Systems," Harbir Lamba also 336 investigated herding behaviors in financial markets. However, instead of using a 337 network model, he proposed a stochastic particle system where each particle is 338 an agent and agents do not interact with each other. Agents' herding behavior is 339 controlled by a herding parameter $C$, which drives the agents' states toward the 340 market sentiment. Using this system, Lamba demonstrated that even a very low 341 level of herding pressure can cause a financial market to transition to a multi-year 342 boom-and-bust.

At time $t$, each agent $i$ in the system can be in one of two possible states, 344 owning the asset $(+1)$ or not owning the asset $(-1)$, according to its pricing strategy 345 $\left[L_{i}(t), U_{i}(t)\right]$. When the asset market price $r_{t}$ falls outside the interval of $L_{i}(t) 346$ and $U_{i}(t)$, agent $i$ switches its state to the opposite state. In addition, when an 347 agent's state is different from the state of the majority agents, its pricing strategy 348 is updated at a rate of $C|\sigma|$, where $\sigma$ is the market sentiment, defined as the average 349 state of all agents. Hence, agents have a tendency to evolve toward the state of the 350 majority agents. Finally, the market price $r_{t}$ is the result of exogenous information 351 and endogenous agent states generated by the agents' evolving pricing strategies. 352

Using 10,000 agents to simulate the market for 40 years, their results showed 353 that even with a low herding parameter value $C=20$, which is much lower than 354 the estimated real market herding pressure of $C=100$, the deviations of market 355 prices away from the equilibrium resemble the characteristics of "boom-and-bust": 356 a multi-year period of low-level endogenous activities that convince equilibrium- 357 believers the system is in an equilibrium state with slowly varying parameters. 358 There then comes a sudden and large reversal involving cascades of agents switching 359 states, triggered by the change in market price. 


\subsection{Macroeconomy}

Product innovation has been shown to play an important role in a firm's perfor- 362 mance, growth, and survival in the modern economy. To understand how product 363 innovation drives the growth of the entire economy, causing business cycle fluctua- 364 tions, in the chapter, entitled "Product Innovation and Macroeconomic Dynamics," 365 Christophre Georges has developed an agent-based macroeconomic model. In this 366 model, a hedonic approach is used, where product characteristics are specified and 367 evaluated against consumer preferences.

368

The macroeconomic environment consists of a single representative consumer 369 and $m$ firms whose products are described by characteristics that the consumer 370 cares about. To satisfy the consumer's utility function, firms improve their product 371 characteristic values through $R \& D$ investment. If the $R \& D$ indeed leads to product 372 innovation that also recovers the cost, the firm grows. Otherwise, the firm becomes 373 insolvent and is replaced by a new firm. 374 A firm can choose to invest or not to invest in R\&D activities. The decision is based 375 on the recent profits of other firms engaging in R\&D and then tuned by the firm's 376 own intensity parameter $\gamma$. When a firm decides to engage in R\&D, the probability 377 that the firm will experience successful product innovation increases. 378

Using 1000 firms and 50 product characteristics to run simulations, the results 379 showed that the evolution of the economy's output (GDP) closely follows the 380 evolution of the R\&D investment spending. Meanwhile, the customer's utility grows 381 over time, due to a long-term net improvement in product quality. Moreover, when 382 the $R \& D$ intensity parameter $\gamma$ is increased, the increased $R \& D$ spending drives up 383 consumption, output, and utility. Finally, ongoing endogenous product innovation 384 leads to ongoing changes in the relative qualities of the goods and the distribution 385 of product shares. The distribution tends to become skewed, with the degree of 386 skewness depending on the opportunities for niching in the product characteristics 387 space. As the number of firms grows large, the economy's business cycle dynamics 388 tends to become dominated by the product innovation cycle of R\&D investment. $\quad 389$

\section{New Methodologies and Technologies for Complex Economic Dynamics}

In addition to the previous ten chapters, this book also includes three chapters, which 392 may not be directly related to agent-based modeling that may provide some useful 393 ideas or tools that can help the modeling, simulation, and analysis of agent-based 394 modeling. We shall also briefly highlight each of them here.

This book is mainly focused on financial markets and market processes. One 396 issue naturally arising is related to how different markets are coupled or connected, 397 and to what degree. In the chapter, entitled "Measuring Market Integration: U.S. 398 Stock and REIT Markets," Douglas Blackburn and N.K. Chidambaran take up 399 
the issue of identifying the existence and extent of financial integration. This is 400 an important methodological issue that empirical studies often encounter, given 401 the complex relationships and heterogeneity that underpins financial markets. The 402 authors identify a potential joint hypothesis problem that past studies testing for 403 financial integration may have suffered from. This problem arises when testing for 404 the equality of risk premia across markets for a common (assumed) set of risk 405 factors; nonetheless, there is a possibility that a conclusion claiming a rejection of 406 integration may actually stem from the markets not sharing a common factor.

407

Overcoming the joint hypothesis problem means disentangling the two issues and 408 examining them separately. They present an approach based on factor analysis and 409 canonical correlation analysis. This approach can be summarized in two steps. First, 410 one should determine the correct factor model in each market and determine whether 411 the markets share a common factor. Second, one should develop economic proxies 412 for the shared common factor and test for the equality of risk premia conditional on 413 a common factor being present. The equality of risk premia is tested only if common 414 factors exist. The authors argue that this procedure in fact gives more power to the 415 tests. They test their method on US REIT and stock markets for 1985-2013. 416

When one attempts to understand social systems as complex systems, for 417 instance, through agent-based models, computers and simulations play a very 418 important role. As the scale and scope of these studies increase, simulations can 419 be highly demanding in terms of data-storage and performance. This is likely 420 to motivate more and more researchers to use highly powerful, supercomputers 421 for their studies as the field matures. In the chapter, entitled "Supercomputer ${ }^{422}$ Technologies in Social Sciences: Existing Experience and Future Perspectives," 423 Valery Makarov and Albert Bakhtizin document several forays into supercomputing 424 in the social science literature.

The authors introduce some open-source platforms that already exist in the 426 scientific community to perform large-scale, parallel computations. They discuss ${ }_{427}$ their hands-on experience in transforming a pre-existing agent-based model into 428 a structure that can be executed on supercomputers. They also present their own 429 valuable experiences and lessons in applying their models to supercomputers. From 430 their experiences, $\mathrm{C}++$ appears to be more efficient than Java for developing soft- 431 wares running on supercomputers. The processes and issues related to translating a 432 Java-based system into a C++ based system are also explained in the chapter. $\quad 433$

Social sciences are distinct from natural sciences in terms of the potential of 434 their theories to have an impact, for better or worse, on the actual lives of people. 435 The great financial crisis of 2008, as some have argued, is a result of over reliance 436 on unrealistic models with a narrow world-view, ignoring the complexities of the 437 financial markets. Should more complex, sophisticated mathematical models be 438 the solution? In the chapter, entitled "Is Risk Quantifiable?", Sami Al-Suwailem, 439 Francisco Doria, and Mahmoud Kamel take up this issue and examine the method- 440 ological issues related to the use of or over-reliance on "formal" models in the social 441 sciences, in particular in economics and finance.

The authors question whether the indeterminacy associated with future economic 443 losses or failures can be accurately modeled and systematically quantified using 444 
formal mathematical systems. Using insights from metamathematics-in particular, 445 Kurt Gödel's famous theorems on incompleteness from the 1930s-they point to the 446 inherent epistemological limits that exist while using formal models. Consequently, 447 they argue that a systematic evaluation or quantification of risk using formal models 448 may remain an unachievable dream. They draw several examples and applications 449 from real-world financial markets to strengthen their argument and the chapter 450 serves as a cautionary message.

\section{Conclusion and Outlook}

Computational economics is a growing field [6]. With the advancement of technolo- 453 gies, modern economies exhibit complex dynamics that demand sophisticated meth- 454 ods to understand. As manifested in this book, agent-based modeling has been used 455 to investigate contemporary financial institutions of dark pools and high-frequency 456 trading (chapters "Dark Pool Usage and Equity Market Volatility", "Modelling 457 Complex Financial Markets Using Real-Time Human-Agent Trading Experiments", 458 and "Does High-Frequency Trading Matter?"). Meanwhile, agent-based modeling 459 is also used to shed light on the market processes or the price discovery processes by 460 examining the roles of traders' characteristics (chapter "Modelling Price Discovery 461 in an Agent Based Model for Agriculture in Luxembourg"), learning schemes 462 (chapters "Heterogeneity, Price Discovery and Inequality in an Agent-Based Scarf 463 Economy" and "Rational Versus Adaptive Expectations in an Agent-Based Model 464 of a Barter Economy"), information exposure (chapter "Does Persistent Learning 465 or Limited Information Matter in Forward Premium Puzzle?"), social networks 466 (chapter "Price Volatility on Investor's Social Network"), and herding pressure ${ }_{467}$ (chapter "The Transition from Brownian Motion to Boom-and-Bust Dynamics in 468 Financial and Economic Systems"). Each of these efforts made is a contribution to 469 enhancing our understanding and awareness of market complexity. Given this extent 470 of complexity, markets may not perform well for many reasons, not just economic 471 ones, but also psychological, behavioral, sociological, cultural, and even humanistic 472 ones. Indeed, market phenomena have constituted an interdisciplinary subject for 473 decades $[11,15,17-19]$. What agent-based modeling can offer is a framework that 474 can integrate these interdisciplinary elements into a coherent body of knowledge. $\quad 475$

Furthermore, agent-based modeling can also help modern economies that have 476 been greatly influenced by the big data phenomenon [7]. By applying computational 477 methods to big data, economists have addressed microeconomic issues in the 478 internet marketplaces, such as pricing and product design. For example, Michael 479 Dinerstein and his co-authors [8] ranked products in response to a consumer's 480 search to decide which sellers get more business as well as the extent of price 481 competition. Susan Athey and Denis Nekipelov [2] modeled advertiser behavior and 482 looked at the impact of algorithm changes on welfare. To work with big data, Google 483 chief economist Hal Varian proposed machine learning tools as new computational 484 methods for econometrics [20]. What will the impact of machine learning be 485 
on economics? "Enormous" answered Susan Athey, Economics of Technology 486 Professor at Stanford Graduate School of Business. "Econometricians will modify 487 the methods and tailor them so that they meet the needs of social scientists primarily 488 interested in conducting inference about causal effects and estimating the impact of 489 counterfactual policies," explained Athey [21]. We also expect the collaborations 490 between computer scientists and econometricians to be productive in the future. 491

Acknowledgements The authors are grateful for the research support in the form of the Taiwan 492 Ministry of Science and Technology grants, MOST 104-2916-I-004-001-Al, 103-2410-H-004- 493 009-MY3, and MOST 104-2811-H-004-003.

\section{References}

1. Arthur, W. B., Holland, J., LeBaron, B., Palmer, R., \& Tayler, P. (1997). Asset pricing under 496 endogenous expectations in an artificial stock market. In W. B. Arthur, S. Durlauf, \& D. Lane 497 (Eds.), The economy as an evolving complex system II (pp. 15-44). Reading, MA: Addison- 498 Wesley.

2. Athey, S., \& Nekipelov, D. (2010). A Structural Model of Sponsored Search Advertising 500 Auctions. Sixth ad auctions workshop (Vol. 15).

3. Barber, B. M., Odean, T., \& Zhu, N. (2009). Systematic noise. Journal of Financial Markets, 502 12(4), 547-569. Amsterdam: Elsevier.

4. Bilson, J. F. O. (1981), The 'speculative efficiency' hypothesis. Journal of Business, 54(3), 504 435-451.

5. Chen, S. H. (2017). Agent-based computational economics: How the idea originated and where 506 it is going. Routledge.

6. Chen, S. H., Kaboudan, M., \& Du, Y.R. (Eds.), (2018). The Oxford handbook of computational 508 economics and finance. Oxford: Oxford University Press. 509

7. Chen, S. H., \& Venkatachalam, R. (2017). Agent-based modelling as a foundation for big data. 510 Journal of Economic Methodology, 24(4), 362-383.

8. Dinerstein, M., Einav, L., Levin, J., \& Sundaresan, N. (2018), Consumer price search and 512 platform design in internet commerce. American Economic Review, 108(7), 1820-59. 513

9. Estola, M. (2017). Newtonian microeconomics: A dynamic extension to neoclassical micro 514 theory. Berlin: Springer.

10. Fama, E. (1984). Forward and spot exchange rates. Journal of Monetary Economics, 14(3), 516 319-338.

11. Halteman, J., \& Noell, E. S. (2012). Reckoning with markets: The role of moral reflection in 518 economics. Oxford: Oxford University Press. 519

12. Jackson, A. (2004). The aggregate behaviour of individual investors, working paper. http://ssrn. 520 com/abstract $=536942$

13. Kumar, A., \& Lee, C. M. C. (2006). Retail investor sentiment and return comovements. Journal 522 of Finance, LXI(5). https://doi.org/10.1111/j.1540-6261.2006.01063.x 523

14. Longworth, D. (1981). Testing the efficiency of the Canadian-U.S. exchange market under the 524 assumption of no risk premium. The Journal of Finance, 36(1), 43-49. 525

15. Lonkila, M. (2011). Networks in the Russian market economy. Basingstoke: Palgrave Macmil- 526 lan.

16. Petrescu, M., Wedow, M., \& Lari, N. (2017). Do dark pools amplify volatility in times of 528 stress? Applied Economics Letters, 24(1), 25-29. 529

17. Rauch, J. E., \& Casella, A. (Eds.), (2001). Networks and markets. New York: Russell Sage 530 Foundation. 
18. Staddon, J. (2012). The malign hand of the markets: The insidious forces on wall street that 532 are destroying financial markets-and what we can do about it. New York: McGraw Hill 533 Professional.

19. Tuckett, D. (2011). Minding the markets: An emotional finance view of financial instability. 535 Berlin: Springer.

20. Varian, H. R. (2014, Spring). Big data: New tricks for econometrics. Journal of Economic 537 Perspectives, 28(2), 3-28.

21. What Will The Impact Of Machine Learning Be On Economics? https://www.forbes.com/.../ 539 what-will-the-impact-of-machine-learning-be-on-economics/), Forbes, Jan 27, 2016.

22. Wolfram, S. (2002). A new kind of science (Vol. 5). Champaign: Wolfram Media. 